\title{
Polyethylene plastic production process
}

\section{Xiuhua Zhong,Xudong Zhao,Yongkang Qian,Yan Zou}

\author{
College of Chemical Engineering, Changzhou University of Technology, Jiangsu, China
}

\begin{abstract}
Polyethylene has become the most important polyolefin plastic with excellent mechanical properties, processing properties and chemical resistance. It is used in the production of film, packaging and pipe. However, the nonpolar property and low rigidity of polyethylene limits its application in certain fields. The new progress of chemical modification, physical modification and modification of polyethylene were reviewed. Chemical modification includes graft modification, copolymerization modification, crosslinking modification, chlorination, chlorosulfonation modification and plasma modification. There are different methods of polyethylene production which include high pressure polyethylene, medium pressure polyethylene and low pressure polyethylene. All three methods have their own benefits and shortcomings which coexist in the industry.

KEYWORDS:polyethylene high pressure production process
\end{abstract}

Citation: Xiuhua Zhong, et al. Polyethylene plastic production process. (2017); 1(1): 1-11.

*Correspondence to: Yan Zou, College of Chemical Engineering, Changzhou University of Technology, Jiangsu, China.38211330011zy@sohu.com

\section{Preface}

The plastics industry is a new industry. Since the mid-nineteenth century, the plastic industry had begun with the introduction of mixture of camphor and nitrocellulose. Since the twentieth century, people had created a series of synthetic resin that is consistent with properties of natural resin using chemical synthesis technique. Since then, the plastics industry begun to flourish and had become a necessary material in many aspects of daily life.

At present, the plastics industry is one of the fastest growing industries in the world. In 1950, the world's plastic production was 1.5 million tons. In 1960, its production had grew to 6.9 million tons. In 1970, it successfully reached 30 million tons. In 1979, its production had doubled to 63.44 million tons. According to foreign forecasts, by 1985 , the world's total output of plastic would rise up to 100 million tons and by 2000 the world's plastic production will be greater than 350 million tons. In the foreseeable future, the world would massively produces quality plastic with volume and weight greater than steel. The future world will be a 'plastic world'.

Polyethylene has excellent resistance to low temperature, great chemical resistance, outstanding power insulation, good resistance to high pressure and marvelous resistance to radiation. Since polyethylene is composed only of carbon and hydrogen, there is no polar element present hence comes with good water resistance.

Polyethylene has different production methods which include high pressure polyethylene, medium pressure polyethylene and low pressure polyethylene. Three methods have their own advantages and disadvantages which coexist in the industry. The properties of polyethylene are different from the manufacturing method and are related to the molecular structure. It can be divided into low and high density.

In general, the polyethylene produced by the high pressure process is called 'low density' and the polyethylene produced by the medium pressure or low pressure process is called 'high density polyethylene'. In addition, there are low molecular weight polyethylene, ultra-high molecular weight polyethylene, cross-linked polyethylene, chlorinated polyethylene, chlorosulfonated polyethylene, ethylene-ethyl acrylate copolymer and other polyethylene and its copolymer. The development of various modification and composite technologies allows polyethylene engage in new applications. 


\section{Introduction to Polyethylene}

\subsection{Introduction}

Polyethylene products is very common in our daily life. For examples, food and pharmaceutical packaging film, wire and cable insulation and pipe. Therefore, the production of polyethylene is humongous as is one of the most popular used polymer materials in day-to-day life. A large number of products could be manufactured from plastic which include plastic bags, plastic film and milk barrels which are suitable for hollow molding, injection molding and extrusion of various products. For instances, various containers, cable cladding, pipe, profile and sheet.

Polyethylene is among the top five world's largest production and consumption of synthetic resin and the main varieties are low-density polyethylene (LDPE), high-density polyethylene (HDPE) and linear low-density polyethylene (LLDPE). In 2002, the production capacity of polyethylene in the world had reached 68,517,000 $\mathrm{t} / \mathrm{a}$, of which Western Europe produced about $20 \%$ of production capacity, about 30\% in North America, Japan accounted for about $5 \%$. Beside Japan, the Asia-Pacific region accounted for about 24\% \%, Africa / Middle East accounted for 12\% whereas Central and South America accounted for about 4\%. In 2001, the global operating rate had dropped to less than $80 \%$ due to the rise in the prices of global production and raw material as a result of economic slowdown. In 2002, as the global economy recovered the polyethylene operating rate rose to around $80 \%$.

Polyethylene is very sensitive to environmental stress (chemical and mechanical) and has poor heat aging resistance. The properties of polyethylene vary depending on the molecular structure and density. The products of different densities ( 0.91 to $0.96 \mathrm{~g} / \mathrm{cm} 3$ ) can be obtained by different production methods. Polyethylene can be processed by general thermoplastics molding methods. They are mainly used to manufacture thin films, containers, pipes, monofilament, wire and cable, daily necessities and is applicable in television, radar and other high-frequency insulation materials. With the development of petrochemical industry, polyethylene production has been developing rapidly, the output of plastic production accounts for about 1 / 4. In 1983 the world's total polyethylene production capacity was $24.65 \mathrm{Mt}$ and had a construction capacity of $3.16 \mathrm{Mt}$.

In recent years, application of polyethylene as a diffusing agent in the field of nuclear physics, astrophysics, reactor operation to measure the amount of neutrons on the nuclear physics was evident.

\subsection{Structure of polyethylene}

Polyethylene (PE) is a type of plastic. The plastic bags that we get from the supermarket are made of polyethylene (PE). Although polyethylene has the simplest structure of the polymer, it is still the most widely used polymer materials. It is made by repeating the - $\mathrm{CH} 2$-unit. Polyethylene is formed by addition polymerization of ethylene $(\mathrm{CH} 2=\mathrm{CH} 2)$. [2]

The performance of polyethylene depends on its polymerization. Ziegler-Natta polymerization was carried out under medium pressure (15-30 atm) organic compound catalytic conditions for high density polyethylene (HDPE). Under these conditions, the polymerized polyethylene molecules are linear and the molecular chain is very long with molecular weight goes up to several hundred thousand. If it is produced under high pressure (100-300MPa), hightemperature (190210C) and in peroxide catalytic conditions free radical polymerization, the end product will low-density polyethylene (LDPE) which is a branched structure.

\section{3. polyethylene in the industry development}

Polyethylene (PE) is the most widely used variety of synthetic resins in China. It is mainly used to make highfrequency insulating materials such as film, container, pipe, monofilament, wire, cable and many other daily necessities. With the development of petrochemical industry, polyethylene production has been rapid development with production accounts for about $1 / 4$ of total plastic production. The rapid expansion China's national economy had created a favorable environment for the development of synthetic resin industry. Polyethylene (PE) industry is expected to grow at a faster rate.

From January to June of 2008, the cumulative production of polyethylene resin was 3,520,250.09 tons which showed an increase of $2.36 \%$ over the same period of last year. From January to June 2008, China imported 2,537,799,893.00 kilograms of primary polymer which worth US $\$ 4,085,020,175.00$, and exports $97,449,745.00$ kilograms of primary polymer which earned RMB 152,849,306.00.

Within the period from 2008 to 2011, new projects in the Asia-Pacific region are mainly located in China, India and South Korea and China. They will continue to be a source of power. China is becoming the world's largest exporter of PE films and bags which supplies to North America, Western Europe and Japan in large quantities. In addition to the industry on the film, woven bags, pipe, cable materials, hollow containers, turnover boxes and other products will lead to strong demand for polyethylene consumption growth. Hence, China's polyethylene production capacity is expected to 
grow rapidly like before. At present, China's polyethylene industry production and development has the following major characteristics:

(1) Rapid increase in production capacity and production;

(2) The supply gap is large, the dependence on imported goods has not changed;

(3) Strong consumer demand in conjunction with the rapid development of the future;

(4) Remains a problem as the industry is becoming increasingly competitive

(5) LLDPE gradually finds its way to the LDPE market share

\subsection{Polyethylene usage}

PE is a promising synthetic material with great physical and chemical properties. PE has high degree of mechanical properties and excellent combination of good dielectric properties. In addition, the molding process is good and prices is low. They are particularly important in following aspects:

(1) Electrical insulation

Due to its high stability, moisture resistance and high dielectric properties, it is an excellent material in the making of insulation material in electrical, non-electrical engineering and many other relevant aspects.

(2) Material to resist chemical agents

Can be used for chemical structure materials such as pipes, anti-corrosion lining and so on.

(3) Packaging

Polyethylene sheet has low density, soft, water impermeable, high tear strength and chemical resistance. These characteristics are necessary for packaging materials hence polyethylene film has a high market value in the packaging industry and is gradually replacing celluloid.

(4) Polyethylene after radiation treatment: $a$ is hard to deform; b will not produce environmental stress cracking; c strong elasticity; d excellent electrical insulation and solvent resistance; e high temperature resistance; f low power factor. Hence the greater performance after radiation puts it into a wider range of uses. For examples, insulating materials for capacitors and transformers and higher temperature parts in aircraft. However, the cross-linking reaction of polyethylene during radiation results in difficulty to undergo subsequent processing.

In addition to the above-mentioned purposes, there are many uses of polyethylene, such as various medical equipment, spraying metal, wood, fabric and other materials. High-density polyethylene can be used as rubber reinforcing agent. [1]

\section{Polyethylene Properties}

\subsection{Polyethylene physical properties}

Ethylene is transparent in the film state but in the presence of massive blocks it is opaque due to the presence of a large number of crystals inside and strong light scattering occurs. The degree of polyethylene crystallization is affected by the number of its branches. The more branches, the more difficult to crystallize. The melting temperature of polyethylene crystal is also affected by the number of branches, distributed in the range from 90 degrees Celsius to 130 degrees Celsius. Polyethylene monocrystals can generally be prepared by dissolving high density polyethylene in xylene at $130{ }^{\circ} \mathrm{C}$ or higher.

Polyethylene is a white waxy translucent material which is soft, tough, lighter than water, non-toxic and has excellent dielectric properties. It is flammable and continue to burn after the fire. Its water permeability is low and the organic vapor transmission rate is larger. The transparency of polyethylene decreases with the increase of crystallinity. Under certain crystallinity, the transparency increases with the increase of molecular weight. High-density polyethylene melting point range of $132-135 \mathrm{oC}$, low-density polyethylene melting point $(112 \mathrm{oC})$ and wide range. At room temperature it is not soluble in any known solvent whereas at $70 \mathrm{oC}$ or greater it can be dissolved in toluene, amyl acetate, trichlorethylene and other solvents.

Polyethylene is odorless, non-toxic, waxy, excellent low temperature performance (minimum temperature up to $-70 \sim-100{ }^{\circ} \mathrm{C}$ ), good chemical stability and has the ability of most of the acid-base erosion (intolerant with oxidative properties of the acid). At room temperature is not soluble in the general solvent. Its water absorption is small but can be slowly dissolved in some organic solvents due to its linear molecules structures. In addition, PE does not swell and has great electrical insulation performance. However polyethylene is very sensitive to environmental stress (chemical 
and mechanical role) and has poor heat resistance. The properties of polyethylene vary depending on the variety which subsequently depends on its molecular structure and density.

\subsection{Polyethylene chemical properties}

Polyethylene has excellent chemical stability. It is resistant to acidic and basic solution such as hydrochloric acid, hydrofluoric acid, phosphoric acid, formic acid, amines, sodium hydroxide and potassium hydroxide at room temperature. However, nitric acid and sulfuric acid has a stronger destructive effect to PE. The polyethylene is susceptible to photo-oxidation, thermal oxidation and ozone decomposition. Under the action of ultraviolet light it is prone to degradation. Carbon on the polyethylene has excellent light shielding effect. After irradiation there will be cross-linking, broken chain and the formation of unsaturated groups and other reflection. Polyethylene is resistant to a variety of organic solvents, resistant to a variety of acid and alkali corrosion but sensitive to antioxidant acids such as nitric acid. In an oxidizing environment, polyethylene is oxidized.

[-CH2-CH2-] $\mathrm{n}$ referred to as PE, ethylene is produced by polymerization of a thermoplastic resin. Copolymers of ethylene with a small amount of alpha-olefins are also included in the industry. Polyethylene is odorless, non-toxic, waxy, excellent low temperature performance (minimum temperature up to $-70 \sim-100^{\circ} \mathrm{C}$ ), good chemical stability and the ability to tolerate most of the acid-base erosion (intolerant with oxidative properties Acid). It is insoluble in general solvent at room temperature, has low water absorption and has excellent electrical insulation performance. However, polyethylene is very sensitive to environmental stress (chemical and mechanical action) and has poor heat aging resistance. The properties of polyethylene vary depending on the variety which subsequently depend on its molecular structure and density. The products of different densities $(0.91$ to $0.96 \mathrm{~g} / \mathrm{cm} 3)$ can be obtained by different production methods. Polyethylene can be processed by general thermoplastics molding methods (see plastic processing). They are mainly used to manufacture thin films, containers, pipes, monofilament, wire and cable, daily necessities, and can be used as television, radar and other high-frequency insulation materials. With the development of petrochemical industry, polyethylene production has been developing rapidly, the output of plastic production accounts for about $1 / 4$. In 1983 the world's total polyethylene production capacity of $24.65 \mathrm{Mt}$, under construction capacity of $3.16 \mathrm{Mt}$.

\subsection{Features of polyethylene}

Its corrosion resistance, electrical insulation (especially high-frequency insulation) is excellent, can be chlorinated easily, chemical cross-linking, irradiation cross-linking modification, available glass fiber reinforced low-pressure polyethylene melting point, great rigidity, hardness and strength, high water absorption, good electrical properties and resistance to radiation. In higher pressure, it has good flexibility, elongation, impact strength and better permeability. High molecular weight polyethylene impact strength and fatigue resistance. Ethylene is suitable for making corrosionresistant parts and insulating parts while high-pressure polyethylene is suitable for making films. Ultra-high molecular weight polyethylene on the other hand is suitable for making shock absorption, wear and transmission parts.

\subsection{Molding characteristics}

(1) Crystalline material, moisture absorption is small, do not need to be fully dry, excellent mobility of the pressure sensitive to the pressure, the use of high pressure in injection molding, temperature uniformity and great filling speed. Note that the choice of gate position is essential to prevent shrinkage and deformation.

(2) Great shrinkage and shrinkage values, prone to deformation warping. Cooling speed should be slow down and a cooling system installed

(3) Heating time should not be too long otherwise it will break down.

(4) Soft plastic parts have a shallow side of the groove, can be forced release.

(5) May melt and hence not to use with organic solvents to prevent cracking

\section{Polyethylene Production Process}

\subsection{Development of polyethylene preparation methods}

In 1933, the British domestic chemical industry found that ethylene can be polymerized under high pressure to produce polyethylene. This method was industrialized in 1939, commonly known as high pressure. In 1953, Federal Republic of Germany K. Ziegler found TiCl4-Al (C2H5) 3 can be used as a catalyst and hence ethylene can be polymerized at lower pressure. This method had brought into the industrial production by the Federal Republic of 
Germany Hurst company in 1955 and referred as low pressure polyethylene. In the early 1950s, Philip Petroleum found the use of chromium oxide - silica gel as a catalyst, ethylene in the pressure can be polymerized to produce high-density polyethylene, and in 1957 to achieve industrial production. In 1960, Canada DuPont began to manufacture low-density polyethylene with ethylene and $\alpha$-olefin solution. In 1977, the United States Carbide Corporation and Dow Chemical Company had made low-pressure method of low-density polyethylene, known as linear low-density polyethylene, which is the United States Carbide's gas phase method. Linear low-density polyethylene and low-density polyethylene have similar performance, but both also have high-density polyethylene characteristics. The combination with low energy consumption in the production gave rise to the rapid development of PE and it has become one of the most compelling new synthetic resin.

The core technology of low pressure is the catalyst. Germany Ziegler invented TiCl4-Al (C2H5) 3 system for the first generation of polyolefin catalyst. The catalytic efficiency is low, about a few grams per gram of polyethylene polyethylene. 1963 Belgium Solvay company pioneered the magnesium compound as the carrier of the second generation of catalyst. The catalytic efficiency of grams per gram of titanium from tens of thousands to hundreds of thousands of grams of polyethylene. The post-treatment step of removing the catalyst residue can also be eliminated by using the second generation catalyst. And later developed a gas-phase efficient catalyst. In 1975, the Italian company was developed to produce granulated polyethylene and direct production of spherical polyethylene catalyst, known as the third generation catalyst which is another milestone for high-density polyethylene production. [4]

\subsection{Production methods}

It could be divided into high pressure method, low pressure method and medium pressure method. High-pressure method is used to produce low-density polyethylene. This method developed early and the use of this production method has accounted for about $2 / 3$ of the total output of polyethylene. However with the production technology and catalyst development, its growth rate has been greatly lagging behind the low pressure method. Low pressure method are slurry method, solution method and gas phase method. The slurry method is mainly used for the production of high density polyethylene, while the solution method and gas phase method can not only produce high density polyethylene, but also by adding comonomer. The production of low density polyethylene is also known as linear low density polyethylene. In recent years, a variety of low-pressure process developed rapidly.

The current process of producing LDPE is mainly by autoclave and high pressure tube method. The use of organic peroxide as initiator and free radical polymerization. Production of polyethylene consist of gas phase, solution, slurry and improved LDPE process. The slurry method is divided into a stirred tank slurry process and a loop reactor slurry method. The LDPE process is the modified version of LDPE equipment production. The pressure will be reduced with the use of Ziegler catalyst production of linear polyethylene. Most of the current LLDPE is produced by gas phase and solution processes, while HDPE is mostly produced by slurry. Traditionally, slurry processes have been used to produce HDPE, but in recent years the application loop reactor in producing LLDPE is also evident.

\subsubsection{High pressure method}

High pressure method uses oxygen or peroxide as initiator as a method in polymerization of ethylene. The ethylene is fed into the reactor by secondary pressure and is polymerized into polyethylene at a pressure of 100 to $300 \mathrm{MPa}$ and at a temperature of 200 to $300^{\circ} \mathrm{C}$ under the action of an initiator. The reaction is separated by pressure and the unreacted ethylene is recovered and recycled. The polyethylene in the addition of plastic additives creates granulation after extrusion.

The polymerization reactor consist of a tubular reactor (pipe length up to $2000 \mathrm{~m}$ ) and a kettle reactor. Tube method has one-way conversion rate of $20 \%$ to $34 \%$, single-line annual production capacity of $100 \mathrm{kt}$. Kettle process flow of one-way conversion rate of $20 \%$ to $25 \%$ while single-line annual production capacity of $180 \mathrm{kt}$.

\subsubsection{Low pressure method}

Low pressure method liquid phase method (also divided into slurry and solution method) and gas phase two, in addition to solution method, the polymerization pressure is below $2 \mathrm{MPa}$. General steps are the preparation of the catalyst, ethylene polymerization, polymer separation and granulation. China's mainly is mainly using Ziegler catalyst slurry method. The most important gas phase eliminates the need for solvent recovery and polymer drying processes, saving $15 \%$ of the investment and $10 \%$ of the operating cost. For the traditional high-pressure method of investment $30 \%$ the operating costs is $1 / 6$. And thus it has been a rapid development. However further improvement show be made for quality and variety in the gas phase.

Conditions and Process Description: Ethylene has a purity of $99 \%$ or more and is polymerized in the presence of catalyst titanium tetrachloride and monochloride diethylaluminum in a gasoline at a pressure of 0.1 to $0.5 \mathrm{MPa}$ and a temperature of 65 to $75 \mathrm{C}$ to obtain a slurry of HDPE. Firstly the destruction of residual catalyst by alcoholysis then 
proceeds with neutralization, washing, and the recovery of gasoline and unpolymerized ethylene. The next step Is drying to obtain granulation product. Features: corrosion resistance, electrical insulation (especially high-frequency insulation) excellent, can be chlorinated. Specific gravity: 0.94-0.96 g / cm ${ }^{3}$ Shrinkage: $1.5-3.6 \%$ Molding temperature: $140-220^{\circ} \mathrm{C}$.

Slurry method- PE produced by this method is insoluble in the solvent. Slurry polymerization conditions are mild and easy to operate. The alkyl aluminum is commonly used as activator and hydrogen as a molecular weight regulator for the use of kettle reactor. The polymer was passed through a flash kettle, a gas-liquid separator to a powder dryer, and then finally granulated. The production process also includes solvent recovery, solvent refining and other steps. Using a different polymerizer in series or in parallel.

It can be divided into two types. First is the mixing tank reactor in which you can obtain different molecular weight distribution products. The second way is slurry method with ring reactor. The former contains two or more reactors, in series or in parallel to produce products with different molecular weights and molecular weight distributions. In the slurry process, monomer, comonomer and hydrogen are dissolved in the diluent. The polymer does not dissolve into the slurry and the reaction temperature and pressure are both low. Due to the polymer does not dissolve the restrictions, it cannot produce low density products but can only produce HDPE. The lower density PE can be produced with lighter isobutane as a diluent (most of which is heavily diluted with other slurries) using Phillipe loop reactor slurry. In recent years manufacturers have used the process to produce LLDPE.

The loop reactor slurry is used in the United States, while Europe and Japan are mostly stirred tank reactors. PhilliPs ring reactor reactor slurry uses chromium oxide as catalyst while the mixing reactor reactor slurry method uses Ziegler Chin catalyst. The molecular weight distribution is narrow. As for the production of high molecular weight HDPE by the use of double-kettle series method, it has reduced productivity and operational control is also more difficult. Slurry industrialization occurs earlier hence its development is more mature and product quality is also better. However, most of the world's HDPE slurry is produced by slurry method. In the United States, slurry production capacity of the entire HDPE production capacity of $2 / 3$. In Western Europe and Japan, the slurry process accounts for a higher proportion of HDPE.

Solution polymerization- Ethylene and polyethylene are dissolved in solvents. The reaction system is homogeneous. Reaction temperature $\left(\geq 140^{\circ} \mathrm{C}\right)$ and pressure $(4 \sim 5 \mathrm{MPa})$ are higher. It is characterized by short polymerization time, high production intensity, high yield, high density, medium and low density of polyethylene which can control the properties of the product better. However, the molecular weight of the polymer obtained by the solution method is low, the molecular weight distribution is narrow while the content is low.

The Dutch DsM stamicarbo craft Dow Process and DuPont process in Canada are relatively important. The first two pressures are lower while the latter pressure is higher. It is also known as medium pressure solution method. The current solution method is used in the medium pressure solution method, the solution process, the monomer and the resulting polymer are soluble in the solvent so the higher the temperature requirements, the pressure is higher. When the polymer molecular weight is high, the solution viscosity, stirring difficulties and production capacity is limited. Subsequently, the production of high molecular weight products becomes more difficult due to the reaction pressure, temperature and polymerization rate.

Ethylene stay for a short time in the reactor and is usually for a few minutes. Switching grade transition time is short and transition material is less. For instance, Canada DuPont pressure solution method stay for Zmin, can be switched between 3 to 4 grades in 24 hours. When the new grade is put into the material, the granulation is only $20 \mathrm{~min}$. The resin produced by the solution method is of good quality and the molecular weight distribution can be very narrow and the melt index can be high. Solution method uses evaporation of solvent recovery method of polymer as energy consumption so that the solvent recovery process is more complex.

Gas phase- Ethylene in the gaseous polymerization uses fluidized bed reactor. The catalyst has two kinds of chromium and titanium. First it takes from the tank and added to the bed with high-speed ethylene cycle to maintain the bed fluidization. Finally it is eliminated by heat polymerization. The resulting polyethylene is discharged from the bottom of the reactor. The reactor pressure is about $2 \mathrm{MPa}$ and the temperature is 85 to $100^{\circ} \mathrm{C}$.

\subsubsection{Medium pressure method}

The medium pressure process uses a chromium catalyst supported on silica gel to polymerize ethylene under medium pressure in a loop reactor to produce high density polyethylene. The ethylene is polymerized under a medium pressure in a loop reactor using a chromium catalyst supported on silica gel to produce high density polyethylene.

\subsection{Production process}

Presently, there are many companies which are specialized in polyethylene technology in the world. There are 7 enterprises with LDPE technology, 10 enterprises with LLDPE and full density technology and 12 enterprises 
with HDPE technology. From the technical development point of view, high-pressure production of LDPE PE resin production technology is the most mature method. Kettle and tube process technology are mature and the current two production technology coexist. Foreign companies generally use low temperature and high activity catalyst initiated polymerization system which can reduce the reaction temperature and pressure.

High pressure production of LDPE will be large-scale and tube-oriented direction. The low pressure production of HDPE and LLDPE uses mainly titanium and network catalyst. Europe and Japan use mainly titanium catalyst whereas United States uses network catalyst.

Catalyst technology: catalyst is a key part of PE technology. In 1991, metallocene catalyst in the United States achieved industrialization which leads to development of PE production technology.

At present, the world's main applications of polyethylene production technology shared 11 species, China's PE production process has 8 kinds:

(1) High pressure tube and kettle reaction process

(2) Mitsui chemical low pressure silt method CX process

(3) BP gas phase Innovene production process

(4) Chevron - Phillips company double loop reactor LPE process

(5) Nordic Chemical North Star (Bastar) Shuangfeng process

(6) Low pressure gas phase Unipol process

(7) Basel Polyolefin Company Hostalen Crafts

(8) Sclartech solution production process

\section{Chapter IV Polyethylene modified varieties}

Modified polyethylene varieties are mainly chlorinated polyethylene, chlorosulfonated polyethylene, cross-linked polyethylene and blended modified varieties.

\subsection{Chlorinated polyethylene}

A chlorine atom obtained by partially replacing the hydrogen atom in the polyethylene with a chlorine moiety. Chlorination is carried out under the action of light or peroxide and is mainly produced by industrial suspension in the industry. Due to the molecular weight of the raw material polyethylene and its distribution, the degree of branching and the degree of chlorination, the distribution of chlorine atoms and the residual crystallinity, it is possible to obtain chlorinated polyethylene of consistency vary from rubbery to hard plastic. The main purpose is to make polyvinyl chloride modifier to improve the impact resistance of PVC. Chlorinated polyethylene itself can also be used as electrical insulation materials and ground materials.

\subsection{Chlorosulfonated polyethylene}

When polyethylene reacts with chlorine containing sulfur dioxide, some of the hydrogen atoms in the molecule are replaced by chlorine and a small amount of sulfonyl chloride $(-\mathrm{SO} 2 \mathrm{Cl})$ groups to give chlorosulfonated polyethylene. The main industrial system is the suspension method. Chlorosulfonated polyethylene is resistant to ozone, resistant to chemical corrosion, oil resistance, heat resistance, light resistance, abrasion resistance and tensile strength. It is a kind of elastomer with good performance and can be used to make contact parts of food.

\subsection{Crosslinked polyethylene}

X-ray, electron beam, ultraviolet radiation or chemical method (peroxide or silicone cross-linking) are employed to make linear polyethylene mesh or body type of cross-linked polyethylene. Silicone cross-linking method is simple, low operating costs and forming and cross-linking can be carried out step by step. Blow molding and injection molding should be adopted. Cross-linked polyethylene is heat resistant, resistant to environmental stress cracking and has better mechanical properties than polyethylene. This makes it suitable for large-scale pipe, cable and rotary molding products. 


\subsection{Blending modified varieties}

The linear low-density polyethylene and low-density polyethylene after blending, can be used for processing films and other products. Polyethylene and ethylene-propylene rubber blends can be used to produce a wide range of thermoplastic elastomers.

\subsection{Metallocene polyethylene}

Metallocene Polyethylene is a novel thermoplastics which resembled the 90's polyolefin industry's most important technological progress following the LLDPE production. Since it uses metallocene (MAO) as polymerization catalyst to produce polyethylene, the performance of the traditional Ziegler-Natta catalyst polymerization in production of $\mathrm{PE}$ is therefore significantly different. Metallocene catalyst in the synthesis of metallocene polyethylene is unique and has excellent performance and application. This had aroused widespread concern in the market and many of the world's leading petrochemical companies had invested huge manpower, material resources competing development and research.

Metallocene catalyst for ethylene polymerization can only be obtained from the molecular weight of 2 to 3 million of the wax and catalytic activity is not as high. There is no practical significance and therefore did not attract attention and promotion. Until 1980, Professor Kaminsky of the University of Hamburg in Germany found that ethylene polymerization was carried out in toluene solution with a co-catalyst combined with zirconocene chloride zirconium dichloride $(\mathrm{CP} 2 \mathrm{ZrCl} 2)$ and methylaluminoxane $(\mathrm{MAO})$ with a catalyst activity of up to $106 \mathrm{~g}-\mathrm{PE} / \mathrm{g}-\mathrm{Zr}$, The reaction rate and enzyme reaction rate. MAO is a high-homogeneous polymethylaluminoxane synthesized by dimethylaluminum and water under conditions other than the polymerization system. Professor Kaminsky's discovery has injected vitality into metallocene catalyst research, attracting many companies involved in development and research and has made considerable progress. In 1991, Exxon (Exxon) achieve the metallocene catalyst for polyolefin industrial production to produce the first batch of metallocene polyethylene (mPE) for the first time. Its trade name is 'Exact'.

The main varieties are linear low density polyethylene (LLDPE) and very low density polyethylene (VLDPE). MPE has two series, the packaging field as the main goal of the film grade and the other is octene- 1 for the comonomer plastic, known as POP (Polyolefine Plastmer). MPE film grades have a lower melting point and a significant melting zone. Its toughness, transparency, hot viscosity, heat sealing temperature and low odor is significantly better than traditional polyethylene in which it can be used to produce heavy bags, metal trash lined, food packaging and stretch film

\section{Polyethylene Processing and Application}

\subsection{Processing and Application}

It can be used in blow molding, extrusion, injection molding and other methods of processing, It is widely used in the manufacture of thin films, hollow products, fiber and daily sundry goods. In the actual production, in order to improve the stability of polyethylene on the oxidation and oxidation, there is the need to add a small amount of plastic additives. Commonly used UV absorbers are o-hydroxybenzophenone or its alkoxy derivatives. Carbon black is an excellent UV shielding agent. In addition, the addition of antioxidants, lubricants and colorants so that the scope of application of polyethylene more expanded.

\subsubsection{Film}

Low-density polyethylene produced by blowing is more than half of the film. This film has good transparency and a certain degree of tensile strength and is widely used in a variety of food, clothing, medicine, fertilizer, industrial packaging materials and Agricultural film. It can also be processed into a composite film extrusion method for packaging heavy objects. Since 1975, high-density polyethylene film has also been developed. It has high strength, low temperature, moisture, good printability and machinability. The use of linear low-density polyethylene is also made of thin film. Its strength and toughness are better than low-density polyethylene. Its puncture resistance and rigidity is also good. Although it has poor transparency, but it is still slightly better than high-density polyethylene. In addition, it can also be processed into paper, aluminum foil or other plastic film extrusion coating polyethylene coating, made of polymer composite materials.

\subsubsection{Hollow products}

High density polyethylene pf high strength is suitable for hollow products. It can be made by blow molding bottles, barrels, cans, tanks and other containers or by casting method into the tank and other large containers. 


\subsubsection{Tubesheet}

Extrusion can produce polyethylene tubing. High density polyethylene pipe strength is greater and is suitable for underground laying. The extruded sheet can be subjected to secondary processing. High-density polyethylene can also be made into foam, foaming and building materials by foaming and foaming.

\subsubsection{Fiber}

China referred it ethylene fiber. Generally it uses low-pressure polyethylene as raw materials which is being spun into synthetic fibers. Ethylene is mainly used for the production of fishing nets and ropes or spun into short fibers for use as flakes. It can also be used for industrial acid and alkali fabric. At present, ultra-high strength polyethylene fiber (strength up to $3 \sim 4 \mathrm{GPa}$ ) has been developed and can be applied as a composite material for bulletproof vest, automobile and offshore operation.

\subsubsection{Sundry goods}

Sundry products produced by injection molding include daily groceries, artificial flowers, turnover boxes, small containers, bicycles and tractor parts.

\section{Conclusions}

The plastics industry is one of the fastest growing industrial sectors in the world and is one of the largest and most versatile thermoplastic general materials. It is made of ethylene poly polymer from the polymer.

There are different production methods which include high pressure polyethylene, medium pressure polyethylene and low pressure polyethylene. The three methods have their own advantages and disadvantages. The properties of polyethylene are different with respect with the different manufacturing method and are also related to the molecular structure. It can be divided into low density and high density.

1. In general, the polyethylene produced by the high pressure process is called 'low density density' and the polyethylene produced by the medium pressure or low pressure process is called 'high density polyethylene'. In addition, there are low molecular weight polyethylene, ultra-high molecular weight polyethylene, cross-linked polyethylene, chlorinated polyethylene, chlorosulfonated polyethylene, ethylene-ethyl acrylate copolymer and other polyethylene and its copolymer. With the development of various modification technologies and composite technologies, polyethylene is engaging into new applications.

\section{$\underline{\text { References }}$}

2. (1) Jin Shao Sheng. Polyethylene row number and processing, Beijing: China Materials Publishing House, 1983.12

3. (2) Fang Du, Jiang Lansun, Wu Zhengde. Ed. Chlor-alkali technology. Chemical Industry Press, 1990

4. (3) Lu Zhongxing, Zhou Yuanpei, ed. Chlor-alkali chemical production process. Chemical Industry Press, 1995.

5. (4) Baidu Encyclopedia. Http: //baike.baidu.com/view/4913.html? Wtp = tt

6. (5) Yao Yuying. Principles of Chemical Engineering. Tianjin Science and Technology Press, 2005-7-1

7. (6) Fu Meili. Oil processing and production technology. Petroleum Industry Press, February 2009 
